\title{
Chlamydia trachomatis samples testing falsely negative in the Aptima Combo 2 test in Finland, 2019
}

Kaisu Rantakokko-Jalava, ${ }^{1,3}$, Kati Hokynar4, Niina Hieta ${ }^{2,3}$, Anniina Keskitalo ${ }^{1,3}$, Pia Jokela5, Anna Muotiala ${ }^{6}$, T. Sakari Jokiranta

Rutta Kuusela ${ }^{8}$, Hannu Sarkkinen', Janne Aittoniemi ${ }^{10}$, Tytti Vuorinen ${ }^{1,3}$, Antti J Hakanen ${ }^{1,3}$, Mirja Puolakkainen ${ }^{5}$

1. Department of Clinical Microbiology, Turku University Hospital, Turku, Finland

2. Venereal Diseases Outpatient Clinic, Turku University Hospital, Turku, Finland

3. University of Turku, Turku, Turku, Finland

4. Department of virology, University of Helsinki, Helsinki, Finland

5. Department of Virology and Immunology, University of Helsinki and Helsinki University Hospital, Huslab, Helsinki, Finland

6. United Medix Laboratories Ltd, Helsinki, Finland

7. Synlab Finland Ltd, Helsinki, Finland

8. Satadiag, Pori, Finland

9. Fimlab, Lahti, Finland

10. Fimlab Laboratories, Tampere, Finland

Correspondence: Kaisu Rantakokko-Jalava (kaisu.rantakokko-jalava@tyks.fi)

Rantakokko-Jalava Kaisu, Hokynar Kati, Hieta Niina, Keskitalo Anniina, Jokela Pia, Muotiala Anna, Jokiranta T. Sakari, Kuusela Rutta, Sarkkinen Hannu, Aittoniemi Janne, Vuorinen Tytti, Hakanen Antti J, Puolakkainen Mirja. Chlamydia trachomatis samples testing falsely negative in the Aptima Combo 2 test in Finland, 2019.

Euro Surveill. 2019;24(22):pii=1900298. https://doi.org/10.2807/1560-7917.ES.2019.24.22.1900298

Since February 2019, over 160 Chlamydia trachomatis (CT) cases testing negative or equivocal by Aptima Combo 2 (AC2) but positive by Aptima CT test run with Panther instruments occurred in Finland. The AC2 test targets chlamydial 23S rRNA while the CT test targets 16S rRNA. Sequencing of 10 strains revealed a nucleotide substitution in 23S rRNA. The significance of this for the failure of the $\mathrm{AC}_{2}$ test to detect the variant is not yet known.

In mid-February, a discrepancy was noticed in testing results for chlamydia in the Clinical Microbiology Laboratory of Turku University Hospital, Turku, Finland. A first-void urine (FVU) sample from a person in their $30 \mathrm{~s}$ had been referred for analysis with a multiplex sexually transmitted infection (STI) assay (Allplex STI Essential, Seegene, Seoul, Korea) and was clearly positive for Chlamydia trachomatis (cycle threshold, Ct 25; cutoff for positivity being $\mathbf{4 0}$ ). A urethral swab specimen taken on the same day was negative by Aptima Combo 2 ( $A C_{2}$ ) test run in the Panther Instrument (Hologic, San Diego, California (CA), United States (US)). The patient had visited the clinic for STI twice in January, and FVU samples on both visits had tested negative in the $\mathrm{AC}_{2}$ test. Moreover, this patient had a chlamydia-positive partner who was tested in another area of the country, where the Abbott m2000 test (Abbott Park, Illinois, US) is used for screening for chlamydia and gonorrhoea.

In the AC2 assay, chlamydial 23SrRNA and/or gonococcal 16SrRNA are amplified by transcription-mediated amplification reaction (TMA), and DNA probes with chemiluminescent label are used to detect the amplicons. The results are given in relative light units (RLU). The amplification of $C$. trachomatis and gonococci can be distinguished by different kinetic profiles. The cutoffs given by the manufacturer are RLU 25 for equivocal ( $R L U \geq 25-<100$ ) and 100 for a positive result $(R L U \geq 100$ ) if only a chlamydial signal is detected. If both chlamydial and gonococcal signals are present or chlamydial result is indeterminate, the cutoff for $C$. trachomatis equivocal is $85 \mathrm{RLU}(\mathrm{RLU} \geq 85-<250)$. Samples with $\geq 250$ RLU are positive for C. trachomatis, if both chlamydial and gonococcal signals are present [1]. As the assay relies on automated equipment (Panther), when a given sample has been processed, the instrument flags the sample as negative, equivocal or positive for chlamydia and/or gonococcus. While the manufacturer's thresholds are accounted for during flagging, the instrument may also take into account other parameters so the results displayed by the instrument may not always reflect an interpretation strictly based on the RLU.

The FVU sample positive in Allplex was tested by $\mathrm{AC}_{2}$ in duplicate and yielded RLUs 25 and 26, interpreted by the instrument as negative for both chlamydia and gonococcus. It was agreed that if there were other patients who had samples flagged negative for $C$. trachomatis despite of clinical suspicion, their samples should be tested also with the multiplex STI assay. During the next week, two such patients were detected (with Allplex test Cts 23 and 35 and AC2 RLUs 23 and 28 , respectively).

As soon as the discrepancy was observed, the distributor of the AC2 test (Immunodiagnostic, Hämeenlinna, 
Samples negative or equivocal for Chlamydia trachomatis by Aptima Combo 2 retested by Aptima CT in Turku, Finland, 11-29 March $2019(\mathrm{n}=943)$

\begin{tabular}{|c|c|c|c|c|c|}
\hline $\begin{array}{l}\text { Qualitative result in the original } \\
\text { AC2 test according to the } \\
\text { instrument display }\end{array}$ & $\begin{array}{c}\text { RLU in the original } \mathrm{AC}_{2} \\
\text { test }\end{array}$ & $\begin{array}{l}\text { Number of samples } \\
\text { tested by ACT test }\end{array}$ & \multicolumn{2}{|c|}{$\begin{array}{c}\text { Positive in the ACT } \\
n \%\end{array}$} & $\begin{array}{l}\text { The } A C 2 \text { test RLU } \\
\text { values in the } A C T \\
\text { positives }\end{array}$ \\
\hline \multirow{4}{*}{$\begin{array}{l}\text { Negative or equivocal for } \mathrm{CT} \\
\text { and negative for } \mathrm{GC}\end{array}$} & $\leq 10$ & 916 & 1 & 0.1 & 3 \\
\hline & $11-15$ & 9 & 1 & 11 & 15 \\
\hline & $16-19$ & 0 & 0 & 0 & n.a. \\
\hline & $20-84$ & 15 & 13 & 87 & $20-49^{a}$ \\
\hline $\begin{array}{l}\text { Negative for } \mathrm{CT} \text { and positive } \\
\text { for GC }\end{array}$ & $>1,300$ & 3 & 0 & 0 & n.a. \\
\hline
\end{tabular}

AC2: Aptima Combo 2; ACT: Aptima CT; CT: Chlamydia trachomatis; GC: Neisseria gonorrhoeae; n.a.: not applicable; RLU: relative light units.

${ }^{\text {a }}$ One sample with RLU 49 was flagged as C. trachomatis equivocal; the others were reported as negative by the Panther instrument.

Finland) rapidly provided us with the Aptima CT (ACT) test kits. The ACT test targets chlamydial 16S rRNA and is recommended by US Centers for Disease Control and Prevention (CDC) guidelines for testing of $C$. trachomatis alone and/or for confirmation of the AC2 test results with an alternative gene target [2]. Since the vast majority of the samples in general yields RLUs $<10$, we decided to retest all samples with $R L U \geq 10$ with the ACT test since mid-February. The first three AC2 false negative samples (respectively from the three patients discussed above) were clearly positive with the ACT test with RLUs>6,000 (cutoffs for low positive 100 and positive 5,000 [3]). During the first week of March, six more false-negative/equivocal patients by $\mathrm{AC}_{2}$ were found positive by ACT.

\section{Case definition for false negative Chlamydia trachomatis and detected cases}

A case was preliminarily defined as a person with an AC2-negative or equivocal sample yielding RLU in the range of $20-84$ (with $R L U<25$ being negative) and a positive result in the ACT test.

Between February and the end April 2019, a total of 26 samples from 25 patients fulfilling these criteria were identified in Turku. Of these, 11 were male FVUs, 12 female FVUs and two cervical swabs. The mean age of patients was 28 years (range: $17-48$ ). Twenty-two samples were flagged in $\mathrm{AC}_{2}$ as negative for C. trachomatis and four as equivocal, three of the latter being requested new samples from patients whose samples had been flagged negative with RLUs 24-34 about 4 weeks earlier. Eighteen of the 26 samples were also tested with Abbott m2000 that amplifies two targets in the cryptic plasmid. All except one were positive (the one sample with Ct 35 in Seegene Allplex test remained negative).

\section{Prevalence of missed Chlamydia trachomatis cases in Southwest Finland}

To assess the prevalence of $A C 2$-negative or equivocal (RLU 20-84)/ACT-positive samples in Turku area (hospital district of Southwest Finland), a total of 943 samples sent for chlamydia testing with negative or equivocal result in the $A C 2$ test were reanalysed by ACT. The percentage of ACT-positive results among the samples with various RLUs in the $A C 2$ test is shown in Table 1.

Among all $C$. trachomatis positive samples by ACT or AC2 or both during the same period, $15 \%$ (15/98) were positive only with the ACT test, including one that was flagged as equivocal in $A C 2$ test. Four of the AC2 negative $(n=3)$ or equivocal $(n=1) / A C T-p o s i t i v e$ samples were from patients who had been recalled for a control specimen because of a suspicious AC2 test results in early 2019. Without this known catch-up diagnostics the AC2-false negative samples would comprise $10 \%(10 / 98)$ of all chlamydia positives during those three weeks.

Samples referred for chlamydia-gonorrhoea screening are routinely not stored in Turku University laboratory. However, 17 samples ( 7 male and 6 female FVUs, as well as 2 vaginal and 2 cervical swabs) that had been sent for analysis with the previously used Anyplex STI-5 genitourinary panel and tested positive for one of those analytes (Mycoplasma genitalium, M. hominis, Ureaplasma urealyticum, U. parvum or Trichomonas vaginalis) were available. The samples were dated from June to December 2018. One male FVU sample taken in late June 2018 was AC2-negative (RLUs of aliquots 22 and 19) but ACT-positive. The patient had previously given a FVU sample in mid-June and its RLU value in the original $A C 2$ run was 31 and flagged as negative.

Since samples with RLUs in the range of 20-84 (AC2 test) were almost always ACT-positive, their proportion 
Samples re-analysed with Aptima CT test in HUSLAB, Finland, 6 March-30 April 2019 (n=757)

\begin{tabular}{|c|c|c|c|c|c|}
\hline $\begin{array}{l}\text { Qualitative result in the original } \mathrm{AC}_{2} \text { test } \\
\text { according to the instrument display }\end{array}$ & $\begin{array}{c}\text { RLU in the original } \mathrm{AC}_{2} \\
\text { test }\end{array}$ & $\begin{array}{l}\text { Number of samples } \\
\text { tested by ACT test }\end{array}$ & \multicolumn{2}{|c|}{$\begin{array}{c}\text { Positive in the ACT } \\
n \%\end{array}$} & $\begin{array}{l}\text { The } A C 2 \text { test RLU } \\
\text { values in the } A C T \\
\text { positives }\end{array}$ \\
\hline \multirow{5}{*}{$\begin{array}{l}\text { Negative or equivocal for } \mathrm{CT} \text { and negative } \\
\text { for GC }\end{array}$} & $\leq 10$ & 330 & 2 & 0.6 & $5-7$ \\
\hline & $11-15$ & 266 & 7 & 2.6 & $14-15$ \\
\hline & $16-19$ & 71 & 13 & 18 & $16-19$ \\
\hline & $20-84$ & 73 & $68^{\mathrm{a}}$ & 93 & $20-46$ \\
\hline & $85-250$ & 3 & $3^{b}$ & 100 & $89-97$ \\
\hline Negative for $\mathrm{CT}$ and positive for $\mathrm{GC}$ & $48-1,492$ & 14 & 1 & 7.1 & 1,492 \\
\hline
\end{tabular}

AC2: Aptima Combo 2; ACT: Aptima CT; CT: Chlamydia trachomatis; GC: Neisseria gonorrhoeae; RLU: relative light units.

a 0 fo samples $\geq 25$ RLU, one sample was flagged as equivocal in the original AC2 test.

${ }^{\mathrm{b}}$ Of three samples with RLU between 85 and 99, three samples were flagged as equivocal in the original AC2 test.

\section{TABLE 3}

Samples re-analysed with Aptima CT test in United Medix Laboratories Ltd, Finland, 1 March-7 May 2019 (n=193)

\begin{tabular}{|c|c|c|c|c|}
\hline RLU in the original $A C 2$ test & $\begin{array}{c}\text { Number of samples tested } \\
\text { by ACT }\end{array}$ & \multicolumn{2}{|c|}{ Positive in the ACT $n \%$} & $\begin{array}{c}\text { The } A C 2 \text { test RLU values in } \\
\text { the } A C T \text { positives }\end{array}$ \\
\hline$\leq 10$ & 51 & 0 & 0 & n.a. \\
\hline $11-15$ & 101 & 2 & 2.0 & 13,14 \\
\hline $16-20$ & 10 & 3 & 30 & $17,18,20$ \\
\hline $21-84$ & $31^{\mathrm{a}}$ & 31 & 100 & $21-33$ \\
\hline $85-250$ & 0 & 0 & 0 & n.a. \\
\hline
\end{tabular}

AC2: Aptima Combo 2; ACT: Aptima Chlamydia trachomatis; n.a.: not applicable; RLU: relative light units.

a All were flagged as negative by the Panther instrument.

of the studied samples was used as an approximation of the prevalence of $\mathrm{AC}_{2}$ negative or equivocal/ ACT positive cases. During the latter half of 2018 , their average proportion was $0.4 \%(38 / 9,472)$ of all tested samples and $6 \%(38 / 624)$ of samples reported as positive for $C$. trachomatis by AC2.

\section{Geographical distribution of cases}

In addition to Turku University Hospital, the AC2 test is used in five other laboratories in Finland. HUSLAB, the largest clinical microbiology laboratory in Finland, serves the hospital district of Helsinki and Uusimaa and hospital district of Kymenlaakso. United Medix Laboratories Ltd and Synlab Finland Ltd provide chlamydia/gonorrhoea tests mainly for private sector clients and Finnish Student Health Service organisation operating in whole Finland but also for several cities in southern and western Finland. Furthermore, the $\mathrm{AC}_{2}$ test is used in local laboratories of the hospital districts of Satakunta and Päijät-Häme. Together these laboratories cover ca $50 \%$ of all chlamydia-gonorrhoea screening performed in Finland.

After the finding of $\mathrm{AC}_{2}$ negative or equivocal/ACTpositive cases, the ACT test was introduced also in these laboratories to enable testing of samples giving RLU values above the background level in the $A_{2} 2$ test as well as to make possible targeted retesting of e.g. samples collected in the STI clinics. The number of samples tested and the numbers of AC2- flagged negative or equivocal/ACT-positive samples in HUSLAB and United Medix Laboratories are presented in Tables 2 and 3. In the local laboratories of Satakunta and Päijät-Häme hospital districts, seven and five ACT positive specimens with RLUs $20-40$ and $13-25$ in the original $A C 2$ test, respectively, have been detected. Synlab Finland Ltd has detected 13 cases. The location of the 


\section{FIGURE}

Numbers of detected AC2-negative or equivocal (RLU 20-84)/ACT-positive cases in hospital districts of Finland, mid-February-April $2019(\mathrm{n}=180)$

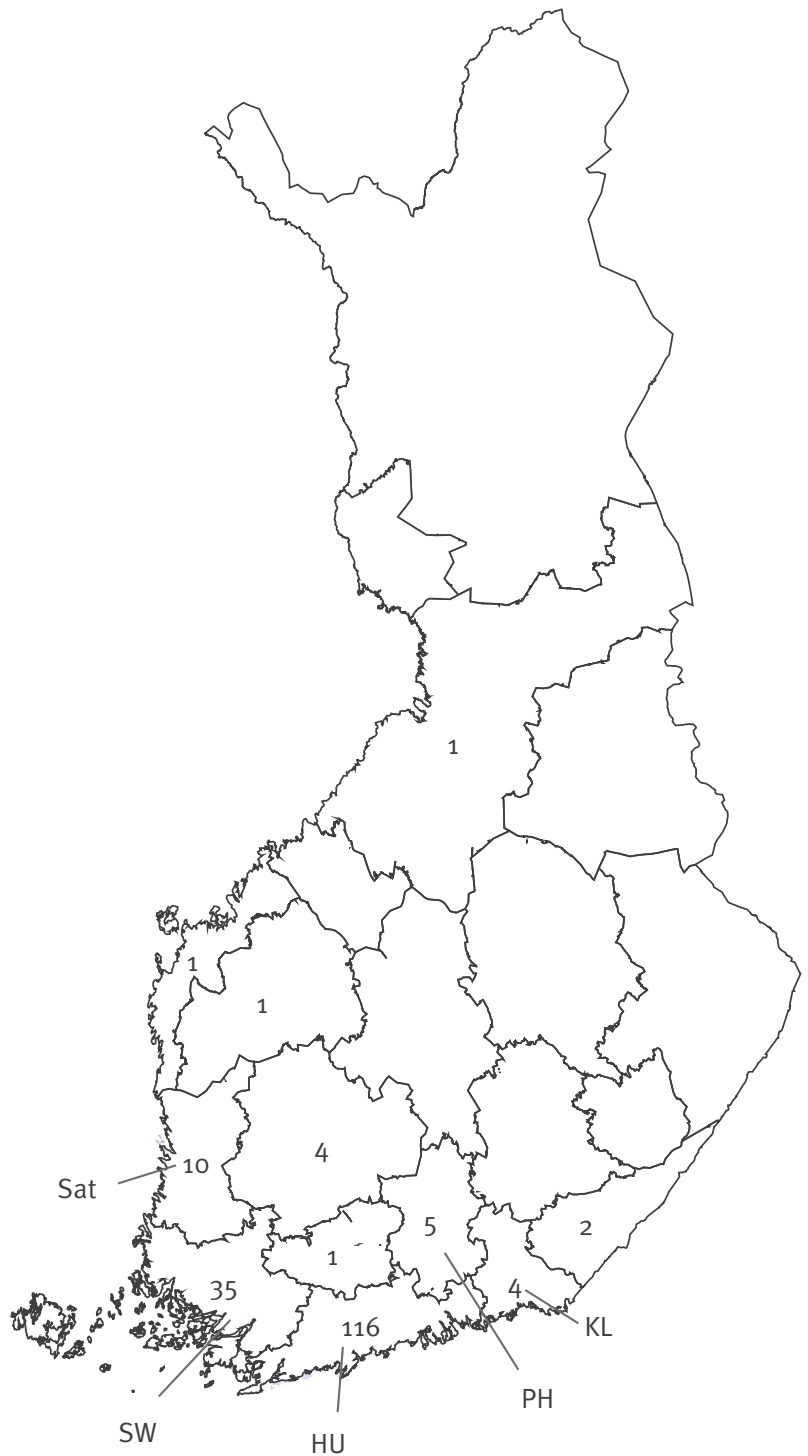

AC2: Aptima Combo 2; ACT: Aptima CT; HU: Helsinki and Uusimaa; KL: Kymenlaakso; PH: Päijät-Häme; RLU: relative light units; Sat: Satakunta; SW: Southwest Finland.

The number of cases found in each district figures for each district on the map. The districts in which both public and private laboratories use Aptima Combo 2 are indicated.

hospital districts where cases have been found and the number of cases in each district is shown in the Figure.

\section{Molecular analysis of cases}

Respective samples from 10 cases, five originating from Turku and five from Helsinki area have been analysed at the University of Helsinki. Initial molecular characterisation (sequencing of $23 \mathrm{~S}$ rRNA, 16S rRNA and typing based on momp gene) of the $A C_{2}$ negative or equivocal (RLU 20-84)/ACT positive specimens show that all are genotype $E$, the most common genotype in Finland [4]. Compared with the reference sequence, $C$. trachomatis E/Bour (HE601870.1), there is a single nt change in the chlamydial 23S rRNA gene in position $1515(C \rightarrow T)$ in the initially falsely negative or equivocal specimens. Thus far, we analysed only a limited number of $A C 2$ negative or equivocal/ACT positive samples $(n=10)$. The sequences have been deposited in GenBank (GenBank accession number MNoo6991*). They all contain the same change, whereas this change cannot be found in the $C$. trachomatis reference strain sequences deposited in GenBank or in our previously sequenced $C$. trachomatis isolates [5] that had been $\mathrm{AC} 2$ positive.

\section{Discussion}

Previously, failure of $C$. trachomatis nucleic acid detection test or tests to detect a variant strain was reported from Sweden. The Swedish new variant of $C$. trachomatis (nvCT) was first described in late 2006 and detected when assessing the reason behind an unexpected $25 \%$ decrease in the number of chlamydia cases in Halland area on the western coast of Sweden [6]. The strain had a 377-bp deletion within its cryptic plasmid that included the only targets of Roche and Abbott CT/GC tests of that time. The individuals with false negative test results remained untreated and unintentionally spread the infection further. The failure of diagnostics had provided the nvCT with a selective advantage, so that by the time of its discovery, nvCt had gained a high proportion (up to 64\%) of all chlamydia cases in some areas where it could spread undetected $[7,8]$. It was estimated that the variant had emerged in 2000-2002, i.e. the spread could have happened in about 5 years by introduction of the strain into high-frequency transmitting populations [8].

Thus far, we have not been able to confirm that the change detected in the $23 \mathrm{~S}$ rDNA is the reason behind the current phenomenon, however it remains a likely explanation. Specific primers and probes are used in the target capture, TMA, and detection steps of the AC2 assay. Even a single change in a suitable location could ruin performance of the test. The manufacturer has excluded erroneous function of the instruments and possibility of improperly functioning lots. A part of discrepant results may be due to higher sensitivity of single target detection in Aptima CT as compared with $\mathrm{AC}_{2}$ according to the performance data in the package inserts or lower sensitivity of the $A C 2$ test in female FVU samples $[1,3,9]$. We do not know which of the patients (if any) detected thus far belong to same transmission chains. It is possible that strains with this change have been present in low numbers for a longer period of time.

Whatever the reason behind the false negative $\mathrm{AC}_{2}$ test results, the finding of $6-10 \%$ more positives by an alternative test reminds us of the general principles in chlamydia testing: no single test detects all cases and the sensitivity of a given method vary according to the sample type. However, also the lessons learned from the Swedish variant are valid and worth a reminder [8]: a single target should not be trusted in molecular 
diagnostics of infections. In our case the extended STI panel fortunately worked as a backup and it probably resulted in the discovery of the variant in an early phase of its spread. The first discrepant case was detected by an alert microbiologist trainee in reporting extended panel samples which are handled more manually than the chlamydia screening samples studied by the highly automated instrument with negative results sent directly to laboratory information system. Also, the close connection between laboratory and STI clinic played a crucial role in the rapid investigation of the discrepancy observed. The traditional quality indicators of chlamydia tests include close follow-up of the percentage of positives, which has not decreased in our laboratories. However, manufacturers of molecular tests should develop algorithms to monitor events and trends in the data produced by their instrument, e.g. percentage of samples in the grey zone. The increased use of molecular systems and automated 'black box automates' challenges us to find new strategies to keep up with the microbial evolution and safeguard the continuous high quality of diagnostics.

\section{*Addendum}

The GenBank accession number was added on 04 June 2019.

\section{Acknowledgments}

The laboratory staff running Panther in Tyks and other laboratories are thanked for the extra work due this investigation. The nurses of Tyks STI clinic are acknowledged for skillful patient work and great cooperation with the laboratory over years. The skillful technical assistance of Anu Kaitonen in PCR is gratefully acknowledged. Thanks are also due to many colleagues who have recalled the patients to give control samples.

\section{Conflict of interest}

None declared.

\section{Authors' contributions}

Kaisu Rantakokko-Jalava: lead of the outbreak investigation, manuscript preparation; Kati Hokynar: sequencing of the variant; Niina Hieta: outbreak investigation, manuscript preparation; Anniina Keskitalo: original observation of the discrepancy and outbreak investigation; Pia Jokela: data from HUSLAB and manuscript preparation; Anna Muotiala: data from United Medix Laboratories Ltd; Sakari Jokiranta: data from Synlab Finland Ltd, map of cases and manuscript preparation; Rutta Kuusela: data from Satadiag; Hannu Sarkkinen: data from Päijät-Häme, Fimlab Lahti, Janne Aittoniemi: confirmation of samples at Fimlab, Tampere; Tytti Vuorinen: outbreak investigation and manuscript preparation; Antti Hakanen: outbreak investigation and manuscript preparation; Mirja Puolakkainen: sequencing, genetics of $\mathrm{C}$. trachomatis and manuscript preparation.

\section{References}

1. Package insert Aptima Combo 2. https://www.hologic.com/ sites/default/files/2019-04/502183EN-IFU-PI_007_01.pdf
2. Boyadzhyan B, Yashina T, Yatabe JH, Patnaik M, Hill CS. Comparison of the APTIMA CT and GC assays with the APTIMA combo 2 assay, the Abbott LCx assay, and direct fluorescentantibody and culture assays for detection of Chlamydia trachomatis and Neisseria gonorrhoeae. J Clin Microbiol. 2004;42(7):3089-93. https://doi.org/10.1128/JCM.42.7.30893093.2004 PMID: 15243065

3. Package insert Aptima CT. https://www.hologic.com/sites/ default/files/2019-04/502184EN-IFU-PI_007_01.pdf

4. Korhonen S, Hiltunen-Back E, Puolakkainen M. Genotyping of Chlamydia trachomatis in rectal and pharyngeal specimens: identification of LGV genotypes in Finland. Sex Transm Infect. 2012;88(6):465-9. https://doi.org/10.1136/ sextrans-2011-050458 PMID: 22517888

5. Hadfield I, Harris SR, Seth-Smith HMB, Parmar S, Andersson P Giffard PM, et al. Comprehensive global genome dynamics of Chlamydia trachomatis show ancient diversification followed by contemporary mixing and recent lineage expansion. Genome Res. 2017;27(7):1220-9. https://doi.org/10.1101/ gr.212647.116 PMID: 28588068

6. Ripa T, Nilsson P. A variant of Chlamydia trachomatis with deletion in cryptic plasmid: implications for use of PCR diagnostic tests. Euro Surveill. 2006;11(11):3076. PMID: 17213548

7. Herrmann B, Törner A, Low N, Klint M, Nilsson A, Velicko I, et al. Emergence and spread of Chlamydia trachomatis variant, Sweden. Emerg Infect Dis. 2008;14(9):1462-5. https://doi. org/10.3201/eid1409.080153 PMID: 18760021

8. Unemo M, Clarke IN. The Swedish new variant of Chlamydia trachomatis. Curr Opin Infect Dis. 2011;24(1):62-9. https://doi. org/10.1097/QCO.ob013e32834204d5 PMID: 21157332

9. Nelson HD, Zakher B, Cantor A, Deagas M, Pappas M. Screening for gonorrhea and chlamydia: systematic review to update the U.S. Preventive Services Task Force Recommendations. Evidence Synthesis, No. 115. Rockville, MD: Agency for Healthcare Research and Quality; September 2014.

License, supplementary material and copyright

This is an open-access article distributed under the terms of the Creative Commons Attribution (CC BY 4.0) Licence. You may share and adapt the material, but must give appropriate credit to the source, provide a link to the licence and indicate if changes were made.

Any supplementary material referenced in the article can be found in the online version.

This article is copyright of the authors or their affiliated institutions, 2019. 